9th A. Friedmann International Seminar and

3rd Casimir Symposium 2015

International Journal of Modern Physics: Conference Series

Vol. 41 (2016) 1660139 (7 pages)

(C) The Author(s)

DOI: $10.1142 /$ S2010194516601393

\title{
Precise digital demodulation for fiber optic interferometer sensors
}

\author{
Andrei Medvedev*, Andrei Berezhnoi, Aleksei Kudryashov and Leonid Liokumovich \\ Institute of Physics, Nanotechnology and Telecommunications, \\ Peter the Great St. Petersburg Polytechnic University, \\ Polytechnicheskaya 29, Saint Petersburg, 195251, Russia \\ *medvedev@rphf.spbstu.ru
}

Received 5 October 2015

Published 18 March 2016

\begin{abstract}
Different methods are used in the interferometer sensors for target signal extraction. Digital technologies provide new opportunities for precise signal detection. We have developed the principle of signal demodulation using an additional harmonic phase modulation and digital signal processing. The principle allows implementation of processing algorithms using different ratios between modulation and discretization frequencies. The expressions allowing calculation of the phase difference using the inverse trigonometric functions were derived. The method was realized in LabVIEW programming environment and was demonstrated for various signal shapes.
\end{abstract}

Keywords: Fiber optic sensor; interferometer; polarization; phase modulation; digital signal processing.

PACS numbers: 42.81.Gs; 07.50.Qx; 07.60.Ly; 07.60.Vg

\section{Introduction}

Fiber-optic interferometers are widely used nowadays along with other devices that utilize the modulation of optic waves parameters by some physical value. This occurs due to the development of laser and fiber-optic technologies as measuring devices. The photodetector output of the interference system is

$$
u(t)=U_{0}+U_{m} \cos \left[\phi_{S}(t)\right]
$$

where $\phi_{S}(t)$ is a target phase difference carrying information about the measured value, $U_{0}$ is a constant component, $U_{m}$ is an interferometer signal amplitude. However, such a signal does not provide means to determine $\phi_{S}(t)$ uniquely. Moreover,

This is an Open Access article published by World Scientific Publishing Company. It is distributed under the terms of the Creative Commons Attribution 4.0 (CC-BY) License. Further distribution of this work is permitted, provided the original work is properly cited. 
the constant component and the interferometer signal amplitude can fluctuate in time. Because of these factors determining of the target phase difference $\phi_{S}(t)$ becomes a complicated task.

The problem of phase demodulation can be solved by applying additional phase modulation and analog processing of the interferometer signal. Many of these methods use harmonic additional modulation. In this case the interference signal acquires the form

$$
u(t)=U_{0}+U_{m} \cos \left[\phi_{S}(t)+\phi_{m} \sin \left(\omega_{m} t\right)\right] .
$$

For the signal given by Eq. (2) analog devices are able to determine the target phase difference by synchronous detecting, summation, differentiating, integrating, etc. A so-called pseudo heterodyne method is another well-known algorithm based on the additional phase modulation. There the signal formed at the photo receiver out-put becomes

$$
u(t)=U_{0}+U_{m} \cos \left[\phi_{S}(t)+\omega_{m} t\right]
$$

To demodulate this signal traditional analog phase metric devices can be utilized.

Digital technologies provide new opportunities to demodulate interferometer signals. ${ }^{1}$ Of course, digital methods are more effective when they are not just a digital implementation of the analog algorithms, ${ }^{2}$ but utilize, for example, calculation of inverse trigonometric functions, ${ }^{3}$ Hilbert transform ${ }^{4}$ and even more complicated procedures, ${ }^{5}$ that cannot be implemented using analog signal processing.

\section{Demodulation Method}

Here we present the results of implementation of previously described digital demodulation algorithm of the fiber interferometer signal using additional phase modulation. ${ }^{6}$ The main concept of this method is that digital processing enables implementation of various mathematical procedures and there is no need to obtain the relatively simple signal like Eq. (3). So interferometer output signal used for processing can be of more complex shape but easier to be obtained in optical scheme.

This method enables implementation of demodulation with various parameters. The essential requirement for this approach is using of signal with shape described by Eq. (2). In this case, $\phi_{S}, U_{0}, U_{m}$ can vary. Oscillation of $\phi_{S}$, bears useful information. Fluctuations of $U_{0}, \mathrm{Um}$ are hindering, but considered slow enough in comparison with the period of the modulating signal.

The basis of the demodulation method is calculating of a single value of the target phase $\phi_{S}$, using three interference signal samples per every modulation period. These samples are taken under different values of the additional phase difference caused by the modulation applied. The necessity of using of three samples can be explained as follows. The function given by Eq. (2) describes the modulated interference signal. It includes three unknown parameters that are supposed to be constant while taking samples: target phase $\phi_{S}, U_{0}$, and $U_{m}$. Let us denote these three 
samples $\left\{u^{(0)}, u^{(1)}, u^{(2)}\right\}$, and values of the modulating phase signal $\left\{\phi^{(0)}, \phi^{(1)}, \phi^{(2)}\right\}$, respectively. Then, the system of equations with these unknown parameters can be written as

$$
\begin{aligned}
& u^{(0)}=U_{0}+U_{m} \cos \left(\phi_{S}+\phi^{(0)}\right), \\
& u^{(1)}=U_{0}+U_{m} \cos \left(\phi_{S}+\phi^{(1)}\right), \\
& u^{(2)}=U_{0}+U_{m} \cos \left(\phi_{S}+\phi^{(2)}\right) .
\end{aligned}
$$

Using trigonometric formulas and denoting $\cos \left[\phi^{(0)}\right]=C_{0}, \cos \left[\phi^{(1)}\right]=C_{1}$, $\cos \left[\phi^{(2)}\right]=C_{2}$ and $\sin \left[\phi^{(0)}\right]=S_{0}, \sin \left[\phi^{(1)}\right]=S_{1}, \sin \left[\phi^{(2)}\right]=S_{2}, U_{0}$ and $U_{m}$ can be excluded. Hence, the formula for a tangent of the target phase is

$$
\tan \phi_{S}=\frac{\left(u^{(0)}-u^{(1)}\right)\left(C_{1}-C_{2}\right)-\left(u^{(1)}-u^{(2)}\right)\left(C_{0}-C_{1}\right)}{\left(u^{(1)}-u^{(2)}\right)\left(S_{1}-S_{0}\right)-\left(u^{(0)}-u^{(1)}\right)\left(S_{2}-S_{1}\right)}=\frac{a}{b},
$$

where

$$
\begin{aligned}
& a=\left(u^{(0)}-u^{(1)}\right)\left(C_{1}-C_{2}\right)-\left(u^{(1)}-u^{(2)}\right)\left(C_{0}-C_{1}\right), \\
& b=\left(u^{(1)}-u^{(2)}\right)\left(S_{1}-S_{0}\right)-\left(u^{(0)}-u^{(1)}\right)\left(S_{2}-S_{1}\right) .
\end{aligned}
$$

In view of the formula for a tangent, the target phase $\phi_{S}$ in the range $[-\pi, \pi]$ can be found using the following equations:

$$
\begin{aligned}
& \phi_{S}=\arctan \left[\frac{a}{b}\right], \text { for } b>0 ; \\
& \phi_{S}=\arctan \left[\frac{a}{b}\right]+\pi \operatorname{sign}(a), \text { for } b<0 .
\end{aligned}
$$

\section{Implementation of the Demodulation Method}

We have carried out a series of experiments to test the demodulation method, using an experimental model of a fiber-optic polarization sensor designed for electric field measurement. ${ }^{7}$ It contained an additional polarization modulator that provided the sine modulation to recover the target signal $\phi_{S}(t)$. The shematic of our experimental setup is shown in Fig. 1. The output signal of this system can be described by Eq. (2), which allows application of the considered demodulation method.

The LabVIEW program controlled the hardware, displayed the results and wrote them to file. An additional modulation signal was formed using 16-bit arbitrary waveform generator NI PXI-5421. Signal parameters were chosen according to the demodulation method protocol. ${ }^{6}$ The additional modulation frequency was $10 \mathrm{kHz}$.

The digitizer NI PXIe-5122 registered the interference signal from the photodetector output. The signal sampling frequency was set in accordance with the number of samples processed per every modulation period. Cases with samples per period $N=3,4$ and 5 were considered. 


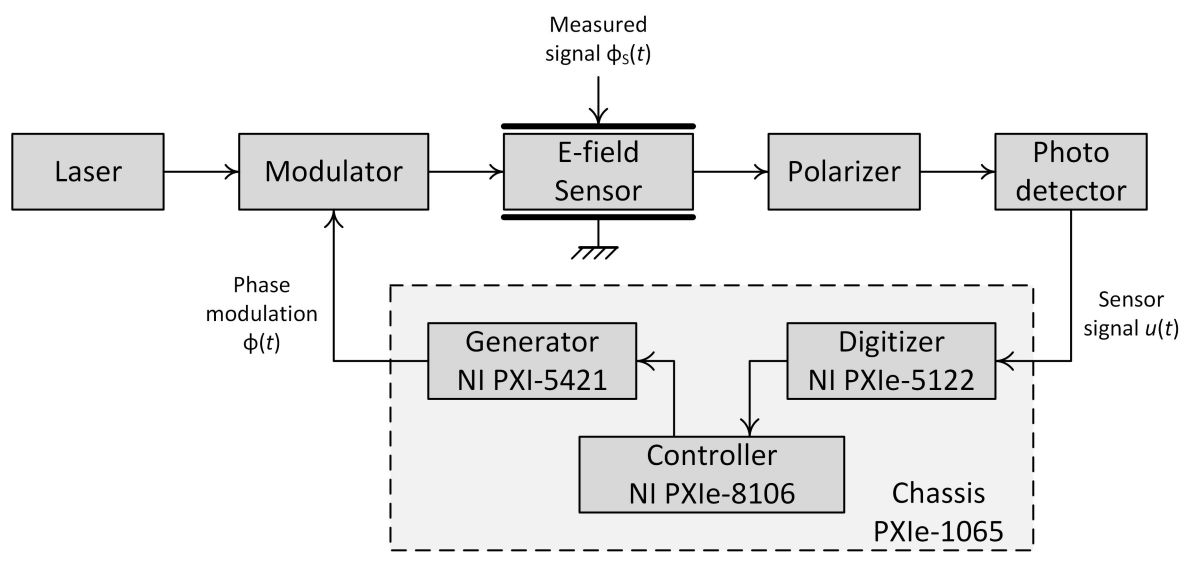

Fig. 1. Experimental setup.

Samples of the additional modulation signal $\phi_{n}$ and of the interference signal $u_{n}$ can be written as follows

$$
\begin{aligned}
\phi_{n} & =\phi\left(t_{n}\right)=\delta \phi_{m} \sin \left[2 \pi f_{M}\left(t_{n}+\Delta t\right)\right]=\delta \phi_{m} \sin \left[\left(\frac{2 \pi n}{N}\right)+\Theta_{0}\right], \\
u_{n} & =u\left(t_{n}\right)=U_{0}+U_{m} \cos \left[\phi_{S}+\phi_{n}\right] \\
& =U_{0}+U_{m} \cos \left[\phi_{S}+\delta \phi_{m} \sin \left\{\left(\frac{2 \pi n}{N}\right)+\Theta_{0}\right\}\right]
\end{aligned}
$$

where $n$ is the sample number starting from the beginning of the first modulation period $(n=0,1,2, \cdots), t_{n}$ is the time of the $n$-th sample, $\Delta t$ and $\Theta_{0}=2 \pi f_{M} \Delta t$ are time delay and a corresponding phase shift of the first sample relatively to the beginning of the first modulation period. In our experiments the sampling sequence was synchronized with the modulating signal without delay $(\Delta t=0)$ regardless of the number of samples $N$ chosen.

Figure 2 shows the acquisition points for different values of $N$ marked on the experimental modulation and interference signals charts.

Acquisition points for the case of $N=3$ are marked with squares. According to the method modulation phase values are

$$
\begin{aligned}
& \phi_{0}=\delta \phi_{m} \sin \left[\left(\frac{2 \pi \cdot 0}{3}\right)+\Theta_{0}\right]=0, \\
& \phi_{1}=\delta \phi_{m} \sin \left[\left(\frac{2 \pi \cdot 1}{3}\right)+\Theta_{0}\right]=\delta \phi_{m} \cdot 0.866, \\
& \phi_{2}=\delta \phi_{m} \sin \left[\left(\frac{2 \pi \cdot 2}{3}\right)+\Theta_{0}\right]=-\delta \phi_{m} \cdot 0.866 .
\end{aligned}
$$




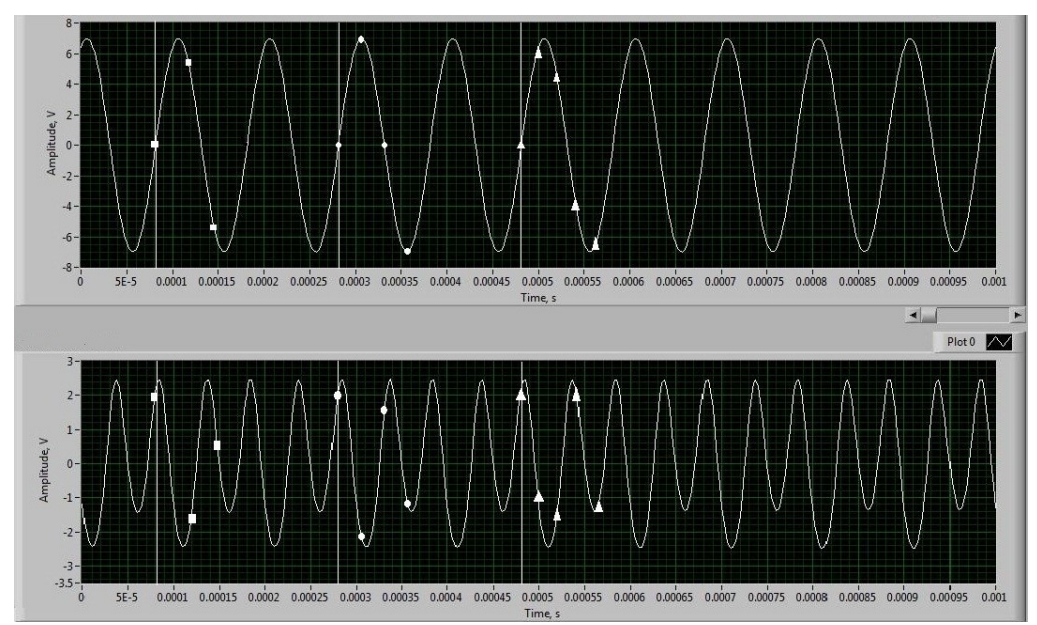

Fig. 2. Acquisition points for the modulation signal (upper trace) and interference signal (lower trace) for different sampling rates.

Acquisition points for the case of $N=4$ are marked with circles. According to the method modulation phase values are

$$
\begin{aligned}
& \phi_{0}=\delta \phi_{m} \sin \left[\left(\frac{2 \pi \cdot 0}{4}\right)+\Theta_{0}\right]=0 \\
& \phi_{1}=\delta \phi_{m} \sin \left[\left(\frac{2 \pi \cdot 1}{4}\right)+\Theta_{0}\right]=\delta \phi_{m} \\
& \phi_{2}=\delta \phi_{m} \sin \left[\left(\frac{2 \pi \cdot 2}{4}\right)+\Theta_{0}\right]=0 \\
& \phi_{3}=\delta \phi_{m} \sin \left[\left(\frac{2 \pi \cdot 3}{4}\right)+\Theta_{0}\right]=-\delta \phi_{m}
\end{aligned}
$$

Acquisition points for the case of $N=5$ are marked with triangles. According to the method modulation phase values are

$$
\begin{aligned}
& \phi_{0}=\delta \phi_{m} \sin \left[\left(\frac{2 \pi \cdot 0}{5}\right)+\Theta_{0}\right]=0 \\
& \phi_{1}=\delta \phi_{m} \sin \left[\left(\frac{2 \pi \cdot 1}{5}\right)+\Theta_{0}\right]=\delta \phi_{m} \cdot 0.95 \\
& \phi_{2}=\delta \phi_{m} \sin \left[\left(\frac{2 \pi \cdot 2}{5}\right)+\Theta_{0}\right]=\delta \phi_{m} \cdot 0.59 \\
& \phi_{3}=\delta \phi_{m} \sin \left[\left(\frac{2 \pi \cdot 3}{5}\right)+\Theta_{0}\right]=-\delta \phi_{m} \cdot 0.59 \\
& \phi_{4}=\delta \phi_{m} \sin \left[\left(\frac{2 \pi \cdot 4}{5}\right)+\Theta_{0}\right]=-\delta \phi_{m} \cdot 0.95
\end{aligned}
$$




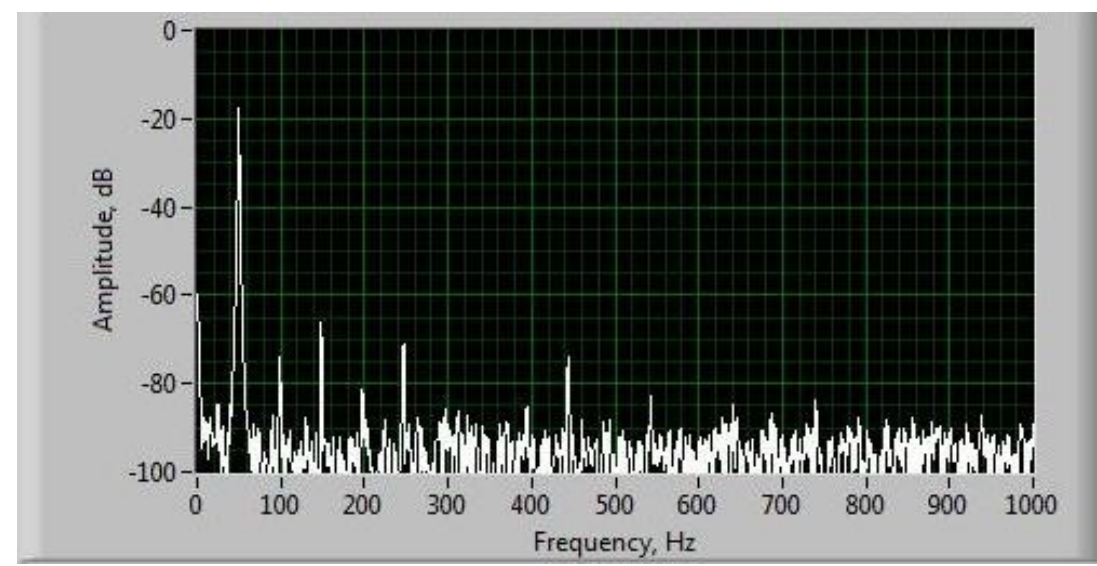

Fig. 3. Spectrum of the sine-shape demodulated signal for $N=3$.

As it was demonstrated in Ref. 6, for the proper calculation of target phase $\phi_{S}$ using triplet of samples $\left\{u^{(0)}, u^{(1)}, u^{(2)}\right\}$, three phase samples $\left\{\phi^{(0)}, \phi^{(1)}, \phi^{(2)}\right\}$ must have different values caused by the additional phase modulation. For example, for the case of $N=4$ modulation triplet $\left\{\phi_{0}, \phi_{1}, \phi_{3}\right\}$ or $\left\{\phi_{1}, \phi_{2}, \phi_{3}\right\}$ with corresponding signal triplet must be picked out for calculation. In the same way, the rule of choosing phase samples can be formulated for other $N$.

For experimental testing of the method, sine, saw tooth and rectangular voltages were applied to a fiber-optic modulator to simulate the measured signal. The frequency of the test voltage was $50 \mathrm{~Hz}$.

The demodulation method was implemented for the number of samples $N=$ $3,4,5$. As the results of the interference signals demodulation, the target signals $\phi_{S}(t)$ were extracted.

The spectrum of the sine-shape demodulated signal is shown in Fig. 3. This spectrum helps to evaluate such parameters as noise level and harmonic distortion. The noise level appears to be $-90 \mathrm{~dB}$, which corresponds to the minimal registered phase shifts of $3 \cdot 10^{-5} \mathrm{rad} / \mathrm{Hz}^{1 / 2}$. The harmonic distortion for the sine signal for $N=3$ was $0.5 \%$.

Similar results were obtained for noise levels and harmonic distortions for other values of $N$ and corresponding optimal modulation amplitudes.

\section{Conclusion}

The paper presents the experimental validation of the method of the interference signal demodulation with additional harmonic phase modulation and digital signal processing in LabVIEW.

The results of the implementation of the processing algorithms for $N=3,4,5$ for various signal shapes demonstrated their effectiveness in application to the interference systems with signals described by Eq. (2). 


\section{References}

1. B. Griffin and M. J. Connelly, Interferometric fiber optic sensor interrogation system using digital signal processing and synthetic-heterodyne detection, in Proc. 17th International Conference on Optical Fibre Sensors (2005) SPIE 5855, p. 619.

2. Nan Zhang, Zhou Meng, Wei Rao and Shuidong Xiong, Investigation on upper limit of dynamic range of fiber optic interferometric sensors base on the digital heterodyne demodulation scheme, in Proc. 22nd International Conference on Optical Fiber Sensors (2012) SPIE 8421, p. 8421BE-28421-BE4.

3. A. C. Cekorich and J. G. Davis, Demodulator and method for interferometric outputs of increased accuracy. US Patent 6,556,509 B1 (2003).

4. L. C. Giulianelli, A. B. Buckman, R. M. Walser and M. F. Becker, Digital demodulation scheme for wide-dynamic-range measurements with a fiber optic interferometer, in Proc. Smart Structures and Materials 1994: Smart Sensing, Processing, and Instrumentation (1994) SPIE 2191, p. 314.

5. A. B. Lewis and S. Russell, Method and apparatus for acoustic sensing using multiple optical pulses. US Patent 2015/0160092A1 (2015).

6. A. V. Kudryashov, L. B. Liokumovich and A. V. Medvedev, Optical Memory and Neural Networks (Information Optics) 22, 236 (2013).

7. L. B. Liokumovich, A. V. Medvedev and V. M. Petrov, Optical Memory and Neural Networks (Information Optics) 22, 21 (2013). 